\title{
Configurational balances via variational arguments
}

\author{
P. PODIO-Guidugli ${ }^{\dagger}$ \\ Dipartimento di Ingegneria Civile, Università di Roma 'Tor Vergata', Via di Tor Vergata 110, \\ I-00133 Roma, Italy
}

[Received 3 January 2000 and in revised form 31 May 2000]

\begin{abstract}
A simple variational argument is presented, yielding the balances of configurational forces both in bulk and at a singular surface in the context of finite elasticity. It is shown that the former balance is equivalent to the bulk balance of standard forces and that the latter balance has instead a physical content which is partly independent of the balance of standard forces at the singular surface.
\end{abstract}

Keywords: Configurational forces; Eshelby stress

\section{Introduction}

'Configurational forces are related to the integrity of a body's material structure and perform work in the transfer of material and the evolution of material structures such as defects and phase interfaces.' ([9], p. 9). These forces and their balance laws play a central role in describing the dynamics of such physical systems as solid/solid phase interfaces, solidification fronts and cracks.

In this paper I present a variational argument that, in statics, yields the balance of configurational forces for an elastic body, both in bulk and at a singular surface. On the way, I illustrate similarities and differences with the classic variational argument yielding the corresponding bulk and jump balances of standard forces. I briefly touch on three concepts-two in continuum mechanics, the force on a defect and the Gibbs function, and one in analysis, the Noether theorem-all related to the Eshelby tensor, the stress measure that enters into the formulation of configurational balances.

The question whether configurational and standard balances are just different forms of statements having the same physical content has been asked ever since configurational forces and their balances where first introduced-in an essentially variational manner-by Eshelby in his pioneering work of the 1950s [3-5]. Although it is clear today to all knowledgeable researchers in continuum physics and materials science that the answer is generally negative (which makes for the interest in configurational forces both from the foundational point of view and in applications), the question is still often asked.

This paper is an updated version of an earlier educational manuscript [15]. In the simplest context of finite elasticity and for a discontinuity in the material organization modelled by an immobile, unstructured singular surface, it is shown that the bulk balances of configurational and standard forces are indeed equivalent, but that there is more to the jump balance of configurational forces than a rephrasing of the jump balance of standard forces: the additional information is carried by the so-called Maxwell relation at the singular surface. The reason for this is to be found in the different types of variations of the energy functional that yield the two sets of balances: while standard balances follow from standard variations of the current shape of the body, leaving the

\footnotetext{
†Email: ppg@uniroma2.it
} 
reference shape fixed, configurational balances follow from the stationarity of the energy functional with respect to variations of the reference shape, leaving the current shape fixed.

The Maxwell relation describes, in the reference shape, the equilibrium of an unstructured singular surface as is determined by the adjacent parts of the body; the variations used mimic a virtual motion of the separating surface due to growth of one part at the expenses of the other. When the separating surface is given thermomechanical structure, other terms may enter the equilibrium relation through a variational approach. Yet no variational principle can handle dynamical situations with general forms of dissipation. The Maxwell relation, however generalized, represents the static version of a balance law for configurational forces that cannot be derived as a stationarity condition [9].

\section{Preliminaries}

Let $\boldsymbol{X}$ be the typical point of a regularly open, bounded region $\Omega$ of $\mathbb{R}^{3}$, the reference placement of a continuous body that for convenience we identify pointwise with $\Omega$; and let $D$ denote the gradient operator. For $f(\boldsymbol{X})$ a deformation of $\Omega$, we write $\boldsymbol{F}$ for $D_{X} f, g(\boldsymbol{x})$ for the inverse of $f$ at $\boldsymbol{x}=f(\boldsymbol{X})$, and $\boldsymbol{G}$ for $D_{x} g=\boldsymbol{F}^{-1}$, and we note that

$$
G F=1, \quad F G=\overline{1},
$$

where $\mathbf{1}$ and $\overline{\mathbf{1}}$ are the identity mappings in the reference placement $\Omega$ and in the current placement $\bar{\Omega}=f(\Omega)$. For $\zeta^{i}$ a system of convected curvilinear coordinates, $\boldsymbol{F}$ and $\boldsymbol{G}$ can be represented as $\boldsymbol{F}=\overline{\boldsymbol{e}}_{i} \otimes \boldsymbol{e}^{i}, \boldsymbol{G}=\boldsymbol{e}_{i} \otimes \overline{\boldsymbol{e}}^{i}$, where $\boldsymbol{e}_{i}=\boldsymbol{X},{ }_{i}$ and $\boldsymbol{e}^{i}=D_{X} \zeta^{i}$ are, respectively, the covariant and contravariant base vectors in the reference copy Ref of the Euclidean three-space where $\Omega$ is placed, while $\overline{\boldsymbol{e}}_{i}=x,{ }_{i}$ and $\overline{\boldsymbol{e}}^{i}=D_{x} \zeta^{i}$ are the corresponding base vectors for the copy $C u r$ where $\bar{\Omega}$ is placed. These representations easily yield, among other things, the relations (2.1), where it is understood that $\mathbf{1}=\boldsymbol{e}_{i} \otimes \boldsymbol{e}^{i}, \overline{\mathbf{1}}=\overline{\boldsymbol{e}}_{i} \otimes \overline{\boldsymbol{e}}^{i}$. We also set $\boldsymbol{F}^{*}:=(\operatorname{det} \boldsymbol{F}) \boldsymbol{F}^{-T}$, so that

$$
\boldsymbol{F}^{*}=(\operatorname{det} \boldsymbol{G})^{-1} \boldsymbol{G}^{T}, \quad \boldsymbol{G}^{*}=(\operatorname{det} \boldsymbol{F})^{-1} \boldsymbol{F}^{T}, \quad \boldsymbol{G}^{*} \boldsymbol{F}^{*}=\mathbf{1}, \quad \boldsymbol{F}^{*} \boldsymbol{G}^{*}=\overline{\mathbf{1}} .
$$

We introduce $\sigma(\boldsymbol{a}, \boldsymbol{b}, \boldsymbol{A})$, a function on $\mathbb{R}^{3} \times \mathbb{R}^{3} \times$ Lin, and interpret

$$
\hat{\sigma}(\boldsymbol{X})=\sigma(\boldsymbol{X}, f(\boldsymbol{X}), \boldsymbol{F}(\boldsymbol{X})), \quad \boldsymbol{X} \in \Omega,
$$

as the stored energy per unit volume of the reference placement when the elastic body $\Omega$ undergoes the deformation $f$. Moreover, we define

$$
\bar{\sigma}(\boldsymbol{a}, \boldsymbol{b}, \boldsymbol{A}):=(\operatorname{det} \boldsymbol{A}) \sigma\left(\boldsymbol{b}, \boldsymbol{a}, \boldsymbol{A}^{-1}\right),
$$

and interpret

$$
\check{\sigma}(\boldsymbol{x}):=\bar{\sigma}(\boldsymbol{x}, g(\boldsymbol{x}), \boldsymbol{G}(\boldsymbol{x})), \quad \boldsymbol{x} \in \bar{\Omega},
$$

as the stored energy per unit volume of the current placement.

We shall be using the stress measures

$$
S:=\partial_{3} \sigma
$$


(the Piola stress; $\partial_{i}$ denotes differentiation with respect to the $i$ th argument, keeping the other arguments fixed), and

$$
\boldsymbol{B}:=\partial_{3} \bar{\sigma}
$$

(no name!) and we shall discuss the role and the interpretation of the Eshelby tensor

$$
\boldsymbol{C}=\sigma \mathbf{1}-\boldsymbol{F}^{T} \boldsymbol{S}
$$

(see [3-5] and references therein; see also [10] and [18]). Eshelby called energy-momentum tensor the construct

$$
\widetilde{\boldsymbol{C}}:=\sigma \mathbf{1}-\boldsymbol{H}^{T} \boldsymbol{S}=\boldsymbol{C}-\boldsymbol{S},
$$

where $\boldsymbol{H}=D_{X} \boldsymbol{u}$ is the gradient of the displacement field $\boldsymbol{u}(\boldsymbol{X}):=f(\boldsymbol{X})-\boldsymbol{X}$.)

As is well known, the Cauchy stress $\boldsymbol{T}$ is related to the Piola stress by the formulae

$$
T=S G^{*}, \quad S=T F^{*} .
$$

Similarly, it follows from (2.4) that

$$
\boldsymbol{B}=\boldsymbol{C} \boldsymbol{G}^{*}, \quad \boldsymbol{C}=\boldsymbol{B F ^ { * }}
$$

moreover,

$$
\boldsymbol{T}=\bar{\sigma} \overline{\mathbf{1}}-\boldsymbol{G}^{T} \boldsymbol{B} .
$$

(Note that switching the reference and current placements induces a one-to-one switch of the elements occupying the same place in the lists $\left\{\boldsymbol{F}, \boldsymbol{F}^{*}, \boldsymbol{S}, \boldsymbol{C}\right\}$ and $\left\{\boldsymbol{G}, \boldsymbol{G}^{*}, \boldsymbol{B}, \boldsymbol{T}\right\}$.)

\section{The Eshelby operators}

Let a singular surface $\mathcal{S}$ exist in the reference placement of the continuous body $\Omega$. In a typical application, $\mathcal{S}$ is interpreted as a sharp, coherent phase interface, and thermomechanical structure is assigned to it, but here such complications are ignored. It is enough to assume that $\mathcal{S}$ is a smooth, oriented surface, that all deformations are continuous across $\mathcal{S}$, and that the deformation gradient, the stored energy and the stress measures are smooth up to $\mathcal{S}$ from either side, although each of these fields may suffer a jump discontinuity across $\mathcal{S}$.

Consider the energy functional

$$
\mathcal{L}\{f\}=\int_{\Omega} \sigma\left(\boldsymbol{X}, f(\boldsymbol{X}), D_{X} f(\boldsymbol{X})\right) \mathrm{d} v
$$

and a standard class of smooth variations $\boldsymbol{h}(\boldsymbol{X})$ that vanish near the boundary $\partial \Omega$ of $\Omega$. (A vector field defined over the closure of $\Omega$ is said to vanish near $\partial \Omega$ if it vanishes over the intersection of the closure of $\Omega$ with a neighbourhood of $\partial \Omega$ [6: Section 8].) Then,

$$
\delta \mathcal{L}\{f\}[\boldsymbol{h}]=\left(\frac{\mathrm{d}}{\mathrm{d} \varepsilon} \mathcal{L}\{f+\varepsilon \boldsymbol{h}\}\right)_{\varepsilon=0}=\int_{\Omega} \boldsymbol{l} \cdot \boldsymbol{h} \mathrm{d} v+\int_{\mathcal{S}} \boldsymbol{l}^{\prime} \cdot \boldsymbol{h} \mathrm{d} a,
$$


where the Euler-Lagrange operator associated to $\mathcal{L}$ consists of two parts $\boldsymbol{l}$ and $\boldsymbol{l}^{\prime}$, namely

$$
\boldsymbol{l}:=\partial_{2} \sigma-\operatorname{Div} \boldsymbol{S}
$$

in bulk (that is, away from $\mathcal{S}$ ) and

$$
\boldsymbol{l}^{\prime}:=[S] \boldsymbol{\nu}
$$

on the singular surface (here $\boldsymbol{\nu}$ is the continuous unit normal field that orients $\mathcal{S}$ ).

If we now define the Eshelby operator in bulk to be

$$
\boldsymbol{m}:=\partial_{1} \sigma-\operatorname{Div} \boldsymbol{C},
$$

it is not difficult to verify the identity

$$
\boldsymbol{m}+\boldsymbol{F}^{T} \boldsymbol{l}=\mathbf{0}
$$

(see formula (2.3) of Ericksen [2]). An important consequence of (3.6) is that, if $f$ is an equilibrium deformation, i.e. if the functional (3.1) is stationary at $f$ and hence the balance of standard forces in bulk

$$
l=\mathbf{0}
$$

holds at $f$, then the balance of configurational forces in bulk

$$
\boldsymbol{m}=\mathbf{0}
$$

holds at $f$ as well (and conversely). Thus,

within a purely variational framework appropriate for elastostatics, the balances of standard and configurational forces in bulk may be regarded as consequences of one and the same physical requirement, namely, the stationarity of the energy integral at equilibria.

(This is perhaps the right place to throw in some semantics. In a fully variational approach such as the one I am using, the concept of force is of course not primitive, be a force standard (a somewhat psychological, subjective qualifier) or configurational (a qualifier used for historical reasons, to which some authors prefer material); in addition, a force balance is nothing more than a stationarity condition. An expression like 'standard force balance' is ambiguous, because it can be unfolded equally well both as 'balance of standard forces' ( $\equiv$ standard-force balance) and as 'standard balance of forces' (三 standard force-balance): after all, there are stationarity conditions that appear to be more standard than others. However, the second alternative would leave room for awkward constructs like 'standard balance of configurational forces'; this is why I prefer the first.)

Note that the stationarity of the functional (3.1) implies, besides the bulk balance (3.7) of standard forces, the jump condition

$$
[S] \boldsymbol{\nu}=\mathbf{0} \text { on } \mathcal{S}
$$

a relation that expresses the balance of standard forces at the singular surface.

At this point one may ask whether $\boldsymbol{m}$ is the bulk part of the Euler-Lagrange operator of some functional and, in the affirmative case, what form the associated jump part should have; and whether 
a formally stated configurational balance at the singular surface would have a physical meaning independent of (3.9).

With a view towards answering these questions, we introduce the functional

$$
\overline{\mathcal{L}}\{g\}=\int_{\bar{\Omega}} \bar{\sigma}\left(\boldsymbol{x}, g(\boldsymbol{x}), D_{x} g(\boldsymbol{x})\right) \mathrm{d} \bar{v}
$$

(note that, for $g=f^{-1}, \mathcal{L}\{f\}=\overline{\mathcal{L}}\{g\}$ ). When we perform the variation of $\overline{\mathcal{L}}$ with respect to a class of smooth variations $\boldsymbol{v}(\boldsymbol{x})$ that vanish near the boundary $\partial \bar{\Omega}$ of $\bar{\Omega}$, we obtain

$$
\delta \overline{\mathcal{L}}\{g\}[v]=\int_{\bar{\Omega}} \bar{l} \cdot v \mathrm{~d} \bar{v}+\int_{\overline{\mathcal{S}}} \bar{l}^{\prime} \cdot v \mathrm{~d} \bar{a},
$$

where

$$
\overline{\boldsymbol{l}}:=\partial_{2} \bar{\sigma}-\operatorname{div} \boldsymbol{B}, \quad \overline{\boldsymbol{l}}^{\prime}:=[\boldsymbol{B}] \overline{\boldsymbol{\nu}}
$$

and where $\bar{\nu}$ is the unit outward normal to $\overline{\mathcal{S}}$, the current shape of $\mathcal{S}$.

Now, by (2.4),

$$
\partial_{2} \bar{\sigma}(\boldsymbol{b}, \boldsymbol{a}, \boldsymbol{A})=(\operatorname{det} \boldsymbol{A}) \partial_{1} \sigma(\boldsymbol{a}, \boldsymbol{b}, \boldsymbol{A}),
$$

and hence

$$
\partial_{2} \bar{\sigma}=(\operatorname{det} \boldsymbol{F})^{-1} \partial_{1} \sigma .
$$

Moreover, for $\boldsymbol{L}$ a spatial tensor field, we have that

$$
\operatorname{div} \boldsymbol{L}=(\operatorname{det} \boldsymbol{F})^{-1} \operatorname{Div}\left(\boldsymbol{L} \boldsymbol{F}^{*}\right),
$$

and hence, due to the first of (2.10) and the first of (2.2),

$$
\operatorname{div} \boldsymbol{B}=(\operatorname{det} \boldsymbol{F})^{-1} \operatorname{Div} \boldsymbol{C} .
$$

Thus, (3.12), (3.14), and (3.16) imply that

$$
\overline{\boldsymbol{l}}=(\operatorname{det} \boldsymbol{F})^{-1} \boldsymbol{m} .
$$

On the other hand,

$$
\overline{\boldsymbol{\nu}} \mathrm{d} \bar{a}=\boldsymbol{F}^{*} \boldsymbol{\nu} \mathrm{d} a, \quad\left[\boldsymbol{F}^{*}\right] \boldsymbol{\nu}=\mathbf{0} .
$$

(The second of these relations follows from a well known kinematical jump condition due to Hadamard, $[\boldsymbol{F}]=\boldsymbol{a} \otimes \boldsymbol{\nu}$ : Hadamard's relation implies the continuity across $\mathcal{S}$ of the tangential derivatives of $f$-and these determine the cofactor $\boldsymbol{F}^{*}$ of $\boldsymbol{F}$-while $\boldsymbol{a}$ is the amplitude of the jump at $\mathcal{S}$ of the normal derivative $\partial_{\nu} f=\boldsymbol{F} \boldsymbol{\nu}$ of $f$.)

Consequently, with the use also of the second of (2.10), we find that

$$
\overline{\boldsymbol{l}}^{\prime}=\left|\boldsymbol{F}^{*} \boldsymbol{\nu}\right|^{-1} \boldsymbol{m}^{\prime} .
$$


Interestingly, $\boldsymbol{m}$ is proportional to $\overline{\boldsymbol{l}}$ through the volume Jacobian, and $\boldsymbol{m}^{\prime}$ to $\overline{\boldsymbol{l}}^{\prime}$ through the surface Jacobian.

With (3.17), (3.19), the third of (2.2), and the first of (2.10), the varied functional (3.11) can be written as

$$
\delta \mathcal{L}\{f\}[\boldsymbol{w}]=\int_{\Omega} \boldsymbol{m} \cdot \boldsymbol{w} \mathrm{d} v+\int_{\mathcal{S}} \boldsymbol{m}^{\prime} \cdot \boldsymbol{w} \mathrm{d} a, \quad \boldsymbol{m}^{\prime}:=[\boldsymbol{C}] \boldsymbol{\nu},
$$

where $\boldsymbol{w}(\boldsymbol{X})=\boldsymbol{v}(f(\boldsymbol{X}))$. Note that, by definition, a deformation $f$ maps the boundary of $\Omega$ into the boundary of its image $f(\Omega)$ under $f$. Therefore, $\boldsymbol{w}$ vanishes near $\partial \Omega$, because $\boldsymbol{v}$ vanishes near $\partial \bar{\Omega}$.

We conclude that, with respect to the indicated class of variations, the Euler-Lagrange operator associated to the energy functional consists of the pair $\left(\boldsymbol{m}, \boldsymbol{m}^{\prime}\right)$ of Eshelby operators, with the bulk part $\boldsymbol{m}$ as in (3.5) and the jump part $\boldsymbol{m}^{\prime}$ defined in terms of the Eshelby tensor $\boldsymbol{C}$ by the second of (3.20). Formally, the stationarity of this integral implies, besides (3.8) in bulk, the jump condition

$$
[\boldsymbol{C}] \boldsymbol{\nu}=\mathbf{0} \text { on } \mathcal{S} \text {. }
$$

We refer to this last relation as the balance of configurational forces at the singular surface. (Variations of the reference shape that leave the current shape fixed are used in [12] to exploit a mixed variational principle in finite elasticity in the absence of a singular surface. The use of interchanging the roles of the reference and current shapes in elasticity had been indicated by Shield as early as in 1967 [17].) Together,

the configurational force balances (3.8) and (3.21) express the physical requirement that the energy integral be stationary in a virtual motion emanating from the reference placement, the current equilibrium placement being kept fixed.

We have shown that the bulk balances (3.7) and (3.8) are equivalent. It is important to realize that the jump balances (3.9) and (3.21) generally are not.

The continuity at the singular surface of the standard traction $\boldsymbol{S} \boldsymbol{\nu}$ does imply the continuity of the tangential component of the configurational traction $\boldsymbol{C} \boldsymbol{\nu}$. Indeed, at a point of the singular surface $\mathcal{S}$ where the normal is $\boldsymbol{\nu}$, the following relationship between the constructs $[\boldsymbol{C}] \boldsymbol{\nu}$ and $[\boldsymbol{S}] \boldsymbol{\nu}$ holds identically with respect to the choice of the tangent vector $\tau$ :

$$
\tau \cdot[C] \nu=F \tau \cdot[S] \nu
$$

Hence, if (3.9) holds,

$$
\boldsymbol{\tau} \cdot[\boldsymbol{C}] \boldsymbol{\nu}=0 .
$$

The content of (3.21) independent of (3.9) is

$$
\boldsymbol{\nu} \cdot[\boldsymbol{C}] \boldsymbol{\nu}=0:
$$

the normal component of the configurational traction is continuous at the singular surface. Interestingly, (3.24) can be casted in a form called the Maxwell relation:

$$
[\sigma]=[\boldsymbol{F} \boldsymbol{\nu} \cdot \boldsymbol{S} \boldsymbol{\nu}]
$$


Conversely, if (3.21) holds, then (3.22) implies that $\overline{\boldsymbol{\tau}} \cdot[\boldsymbol{S}] \boldsymbol{\nu}=0$ for each vector $\overline{\boldsymbol{\tau}}=\boldsymbol{F} \boldsymbol{\tau}$ tangent to $\overline{\mathcal{S}}$. Hence, in this case, the independent content of (3.9) is, for $\bar{\nu}$ the normal to $\overline{\mathcal{S}}, \overline{\boldsymbol{\nu}} \cdot[\boldsymbol{S}] \boldsymbol{\nu}=0$, an expression of the continuity at the singular surface of the normal component of the standard traction $S \nu$.

The interested reader is referred to $[7,9,10]$ for a number of generalizations and applications that obtain when configurational forces and their balances are considered in a general, nonstrictly-variational context and, moreover, the singular surface $\mathcal{S}$ is given a peculiar kinematic and constitutive structure. Other works having direct relevance, albeit to a variable extent, to the matters treated here are $[1,8,11,13]$.

\section{The Eshelby tensor and related concepts in continuum mechanics and analysis}

\subsection{Eshelby tensor and the force on a a defect}

For $\Pi$ any part ( $\equiv$ subbody) of $\Omega$ that does not intersect $\mathcal{S}$, the bulk balance of configurational forces can be written as

$$
\int_{\Pi} \partial_{1} \sigma \mathrm{d} v-\int_{\partial \Pi} \boldsymbol{C n} \mathrm{d} a=\mathbf{0}, \quad \Pi \subset \Omega .
$$

The accepted interpretation in materials science is that a material inhomogeneity (a 'defect') induces a lattice distortion that is balanced by the configurational tractions described by the Eshelby tensor C:

$-\int_{\Omega} \partial_{1} \sigma d v \equiv$ the body force that makes up for the lattice distortion in the referential part $\Pi$;

$+\int_{\partial \Pi} \boldsymbol{C n} d a \equiv$ the force on the defect embedded into $\Pi$.

\subsection{Eshelby tensor and the Gibbs function}

In a thermomechanical context, the free energy $\Psi:=\epsilon-\eta \theta$, with $\epsilon \equiv$ internal energy, $\eta \equiv$ entropy and $\theta \equiv$ temperature, replaces for the stored energy $\sigma$ in the definition of the Eshelby tensor:

$$
\boldsymbol{C}=\Psi \mathbf{1}-\boldsymbol{F}^{T} \boldsymbol{S} .
$$

For a thermoelastic fluid, in particular, the free energy has the form

$$
\Psi=\phi(\operatorname{det} \boldsymbol{F}, \theta)-\eta \theta,
$$

the Piola stress is

$$
S=\left(\partial_{1} \phi\right) \boldsymbol{F}^{*},
$$

and the Cauchy stress is a pressure

$$
\boldsymbol{T}=-\pi \overline{\mathbf{1}}, \quad \pi:=-\partial_{1} \phi
$$

(see (2.6) and the first of (2.9), respectively). Consequently, the Eshelby tensor takes the form

$$
\boldsymbol{C}=\psi \mathbf{1}, \quad \psi:=\Psi+\pi \operatorname{det} \boldsymbol{F} \equiv \text { Gibbs chemical potential. }
$$

( $\psi$ is also called the free enthalpy.) Thus, for materials of arbitrary response symmetry, the Eshelby tensor may be seen as a generalization of the scalar chemical potential of fluids ( [18], p. 280). One may argue that a tensorial concept is needed whenever the material response depends on orientation, as is generally the case for materials that are not fluids. In fact, whenever the stress is a pressure, the Eshelby tensor takes the form (4.6) 1 in all materials. 


\subsection{Eshelby tensor and the Noether Theorem}

Recall from Section 2 that Ref and Cur denote two copies of the Euclidean three-space where the continuous body $\Omega$ is placed. Consider an $s$-parameter group $\mathcal{G}$ of Lie transformations of the Cartesian product $\operatorname{Ref} \times C$ ur of the reference and current spaces into itself:

$$
Y=Y(X, x ; \epsilon), \quad y=y(X, x ; \epsilon),
$$

such that

$$
Y(X, x ; 0)=X, \quad y(X, x ; 0)=x,
$$

and let

$$
\boldsymbol{h}=\partial_{\epsilon} \boldsymbol{Y}_{\mid \epsilon=0}, \quad \boldsymbol{g}=\partial_{\epsilon} \boldsymbol{y}_{\mid \epsilon=0}
$$

be the infinitesimal generators of $\mathcal{G}$.

Given a deformation $f$ of $\Omega$, we have from the first of (4.7) that, for each fixed parameter-vector $\boldsymbol{\epsilon}$, the mapping

$$
\overline{\boldsymbol{Y}}(\boldsymbol{X}, \boldsymbol{\epsilon}):=\boldsymbol{Y}(\boldsymbol{X}, f(\boldsymbol{X}) ; \boldsymbol{\epsilon})
$$

has an inverse $\overline{\boldsymbol{X}}(\boldsymbol{Y}, \boldsymbol{\epsilon})$. Thus, we may use the second of (4.7) to define the mapping

$$
\overline{\boldsymbol{y}}(\boldsymbol{Y}, \boldsymbol{\epsilon}):=\boldsymbol{y}(\overline{\boldsymbol{X}}, f(\overline{\boldsymbol{X}}(\boldsymbol{Y}, \boldsymbol{\epsilon})) ; \boldsymbol{\epsilon}) .
$$

The integral $\mathcal{L}\{f\}$ is invariant under the action of $\mathcal{G}$ if, for each deformation $f$, for each part $\Pi \subset \Omega$ and for each choice of $\epsilon$, it so happens that

$$
\int_{\Pi(\boldsymbol{\epsilon})} \sigma\left(\boldsymbol{Y}, \overline{\boldsymbol{y}}(\boldsymbol{Y}, \boldsymbol{\epsilon}), D_{Y} \overline{\boldsymbol{y}}(\boldsymbol{Y}, \boldsymbol{\epsilon})\right) \mathrm{d} v(\boldsymbol{Y})=\int_{\Pi} \sigma\left(\boldsymbol{X}, f(\boldsymbol{X}), D_{X} f(\boldsymbol{X})\right) \mathrm{d} v(\boldsymbol{X}),
$$

where $\Pi(\boldsymbol{\epsilon})=\overline{\boldsymbol{Y}}(\Pi, \boldsymbol{\epsilon})$. By localization, (4.12) is equivalent to

$$
\sigma\left(\boldsymbol{Y}, \overline{\boldsymbol{y}}(\boldsymbol{Y}, \boldsymbol{\epsilon}), D_{Y} \overline{\boldsymbol{y}}(\boldsymbol{Y}, \boldsymbol{\epsilon})\right) \operatorname{det}\left(D_{X} \overline{\boldsymbol{Y}}(\boldsymbol{X}, \boldsymbol{\epsilon})\right)=\sigma\left(\boldsymbol{X}, f(\boldsymbol{X}), D_{X} f(\boldsymbol{X})\right),
$$

for $\boldsymbol{Y}=\overline{\boldsymbol{Y}}(\boldsymbol{X}, \boldsymbol{\epsilon})$ and for all $(\boldsymbol{X}, \boldsymbol{\epsilon})$. Thus, the invariance of $\mathcal{L}$ under the $s$-parameter group $\mathcal{G}$ implies that the following $s$ Noetherian identities hold:

$$
D_{X} \sigma \cdot \boldsymbol{h}+D_{X} \sigma \cdot \boldsymbol{g}+D_{F} \sigma \cdot(\operatorname{Grad} \boldsymbol{h}-\boldsymbol{F} \mathrm{Grad} \boldsymbol{g})+\sigma \operatorname{Div} \boldsymbol{h}=0 .
$$

(Here $\boldsymbol{h}=\boldsymbol{h}(\boldsymbol{X}, \boldsymbol{x}(\boldsymbol{X}))$ et sim. One can arrive at (4.14) also through less stringent a notion of invariance; namely, one can term $\mathcal{L}$ infinitesimally invariant under $\mathcal{G}$ whenever $D_{\boldsymbol{\epsilon}}\left(\sigma\left(\boldsymbol{Y}, \overline{\boldsymbol{y}}, D_{Y} \overline{\boldsymbol{y}}\right) \operatorname{det}\left(D_{X} \overline{\boldsymbol{Y}}\right)\right)_{\left.\right|_{\epsilon=0}}=0$.) In view of our previous definitions and, in particular, of (3.6), it is not difficult to write (4.14) in a perspicuous form that features the pair of the Piola stress $S$ and the equilibrium operator $\boldsymbol{l}$, the Eshelby pair $(\boldsymbol{C}, \boldsymbol{m})$ and the pair $(\boldsymbol{h}, \boldsymbol{g})$ of the infinitesimal generators of the symmetry group of the basic integral. This form is

$$
\boldsymbol{l} \cdot \boldsymbol{g}+\boldsymbol{m} \cdot \boldsymbol{h}+\operatorname{Div}\left(\boldsymbol{S}^{T} \boldsymbol{g}+\boldsymbol{C}^{T} \boldsymbol{h}\right)=\mathbf{0} .
$$


From (4.15) we read off the Noether theorem:

let the integral $\mathcal{L}$ be invariant under the action of an s-parameter group $\mathcal{G}$ of Lie transformations of $R e f \times C$ ur in itself. Then, for $\boldsymbol{h}, \boldsymbol{g}$ the infinitesimal generators of $\mathcal{G}$, each deformation that renders $\mathcal{L}$ stationary satisfies also the sonservation laws

$$
\operatorname{Div}\left(\boldsymbol{S}^{T} \boldsymbol{g}+\boldsymbol{C}^{T} \boldsymbol{h}\right)=\mathbf{0} .
$$

(For a thorough discussion in modern terms of this theorem, which was proved by Emmy Noether in 1918, see [14], especially Section 4.4.)

Familiar examples are the one-parameter groups of the translational spatial symmetries:

$$
\begin{cases}\boldsymbol{h}=\mathbf{0}, & \boldsymbol{g}=\boldsymbol{q} \equiv \text { a constant vector } \\ \boldsymbol{Y}=\boldsymbol{X}, & \boldsymbol{y}=\boldsymbol{x}+\varepsilon \boldsymbol{q}\end{cases}
$$

and of the rotational spatial symmetries:

$$
\begin{cases}\boldsymbol{h}=\mathbf{0}, & \boldsymbol{g}=\boldsymbol{W} \boldsymbol{x}, \quad \boldsymbol{W} \equiv \text { a constant skew-symmetric tensor } \\ \boldsymbol{Y}=\boldsymbol{X}, & \boldsymbol{y}=\boldsymbol{x}+(\boldsymbol{Q}(\varepsilon)-\overline{\mathbf{1}}) \boldsymbol{x}, \quad \boldsymbol{Q}(\varepsilon)=\exp (\varepsilon \boldsymbol{W}) .\end{cases}
$$

The conservation laws associated with (4.17) and (4.18) are, respectively,

$$
\operatorname{Div} \boldsymbol{S}=\mathbf{0},
$$

and

$$
\left(\boldsymbol{S F}^{T}-\boldsymbol{x} \otimes \operatorname{Div} \boldsymbol{S}\right) \equiv \text { a symmetric tensor. }
$$

Note that, while equations (3.3) and (3.7) combine to give $\partial_{2} \sigma-\operatorname{Div} \boldsymbol{S}=\mathbf{0}$, translational invariance rules out any dependence of $\sigma$ on its second argument, whence (4.19). Other familiar examples obtain by writing down groups of material symmetries and deducing the associated conservation laws; mixed symmetries are less frequented [16].

To sum up, the typical use of the Noether theorem is as follows. Suppose a hyperelastic constitutive class is chosen together with its presumed symmetries, the latter being specified by an $s$-parameter group of invariance transformations of $R e f \times C u r$ into itself. Necessarily, then, all admissible deformation have to satisfy a system of $s$ identities involving the constitutive mapping and the infinitesimal generators of the group. From these identities, a system of $s$ conservation laws is deduced, which are satisfied by all equilibrium deformations.

\section{Acknowledgements}

This work has been supported by Progetto Cofinanziato 1998 'Modelli Matematici per la Scienza dei Materiali' and by TMR contract FMRX-CT98-0229 'Phase Transitions in Crystalline Solids'.

The notes [15] were prepared in May 1991, elaborated for the CIRM Course 'Recent Advances in Continuum Mechanics' (Trento, January 1997), and further edited for presentation during the 3d Microcolloquium on Microstructures (Pisa, December 1999). I am indebted to Carmine Trimarco for an inclusive list of references. In this connection, I claim no completeness for the reference list given below, and apologize for possible, unintentional omissions. 


\section{REFERENCES}

1. Dascalu, C. \& Maugin, G. A. The thermoelastic material-momentum equation. J. Elast. 39, (1995) 201-212.

2. ERICKSEN, J. L. Special topics in elastostatics. In: YIH, C.-S. (ed), Advances in Applied Mechanics, pp. 189-244. Academic, New York (1977).

3. Eshelby, J. D. The continuum theory of lattice defects. In: Seitz, F. \& Turnbull, D. (eds), Solid State Physics, vol. 3, pp. 79-156. Academic, New York (1956).

4. EshelBY, J. D. Energy relations and the energy-momentum tensor in continuum mechanics. In: Kanninen, M., Adler, W., Rosenfeld, A. \& JAFFe, R. (eds), Inelastic Behavior of Solids, pp. 77-115. McGraw-Hill, New York (1970).

5. Eshelby, J. D. The elastic energy-momentum tensor. J. Elast. 5, (1975) 321-336.

6. Gurtin, M. E. The Linear Theory of Elasticity. (Handbuch der Physik VIa/2). Springer, Berlin (1972).

7. Gurtin, M. E. Thermomechanics of Evolving Phase Boundaries in the Plane. Oxford Science Publications, Oxford (1993).

8. Gurtin, M. E. The nature of configurational forces. Arch. Rational Mech. Anal. 131, (1995) 67-100.

9. Gurtin, M. E. Configurational Forces as Basic Concepts of Continuum Physics. Springer, Berlin (2000).

10. Maugin, G. A. Material Inhomogeneities in Elasticity. Chapman and Hall, London (1993).

11. Maugin, G. A. Material forces: concepts and applications. Appl. Mech. Rev. 48, (1995) 213-245.

12. Maugin, G. A. \& Trimarco, C. Note on a mixed variational principle in finite elasticity. Rend. Mat. Accad. Lincei IX 3, (1992) 69-74.

13. NORRIS, A. N. The energy of a growing elastic surface. Int. J. Solids \& Struct. 16, (1998) 5237-5252.

14. Olver, P. J. Applications of Lie Groups to Differential Equations. Springer, Berlin (1986).

15. Podio-Guidugli, P. The tensor and the operator of Eshelby, unpublished (May 1991).

16. Podio-Guidugli, P. Old and new invariance methods in continuum mechanics. In: IBRAgimov, N. H. \& Mahomed, F. M. (eds), Modern Group Analysis VI, (Proc. Int. Conf. Johannesburg, Jan 1996). New Age International Publishers, New Delhi (1997) pp. 41-52.

17. SHIELD, R. T. Inverse deformation results in finite elasticity. Z. Angew. Math. Phys. 18, (1967) 490-500.

18. ŠILHAVÝ, M. The Mechanics and Thermodynamics of Continuous Media. Springer, Berlin (1997). 